\title{
Prospective evaluation of fexapotide triflutate injection treatment of Grade Group 1 prostate cancer: 4-year results
}

\author{
Neal Shore ${ }^{1}$ (D) Steven A. Kaplan ${ }^{2} \cdot$ Ronald Tutrone $^{3} \cdot$ Richard Levin $^{4} \cdot$ James Bailen $^{5} \cdot$ Alan Hay $^{6} \cdot$ Susan Kalota ${ }^{7}$. \\ Mohamed Bidair ${ }^{8} \cdot$ Sheldon Freedman ${ }^{9} \cdot$ Kenneth Goldberg $^{10} \cdot$ Frederick Snoy $^{11} \cdot$ Jonathan I. Epstein $^{12}$
}

Received: 5 November 2019 / Accepted: 4 February 2020 / Published online: 22 February 2020

(c) The Author(s) 2020

\begin{abstract}
Purpose This study was undertaken to determine the safety and efficacy of fexapotide triflutate (FT) $2.5 \mathrm{mg}$ and $15 \mathrm{mg}$ for the treatment of Grade Group 1 prostate cancer.

Methods Prospective randomized transrectal intraprostatic single injection FT $2.5 \mathrm{mg}(n=49)$, FT $15 \mathrm{mg}(n=48)$ and control active surveillance (AS) $(n=49)$ groups were compared in 146 patients at 28 U.S. sites, with elective AS crossover $(n=18)$ to FT after first follow-up biopsy at 45 days. Patients were followed for 5 years including biopsies (baseline, 45 days, and 18, 36 , and 54 months thereafter), and urological evaluations with PSA every 6 months. Patients with Gleason grade increase or who elected surgical or radiotherapeutic intervention exited the study and were cumulatively included in the data analysis. Percentage of normal biopsies in baseline focus quadrant, tumor grades, and volumes; and outcomes including Gleason grade in entire prostate as well as treated prostate lobe, interventions associated with Gleason grade increase and total incidence of interventions were assessed.

Results Significantly improved long-term clinical outcomes were found after 4-year follow-up, with percentages of patients progressing to interventions with and without Gleason grade increase significantly reduced by FT single treatment. Results in the FT 15-mg group were superior to the FT 2.5-mg dose group. There were no drug-related serious adverse events (SAEs). Conclusions FT showed statistically significant long-term efficacy in the treatment of Grade Group 1 patients regarding clinical and pathological progression. FT $15 \mathrm{mg}$ showed superior results to FT $2.5 \mathrm{mg}$. There were no drug-related SAEs; FT injection was well tolerated.
\end{abstract}

Keywords Prostate cancer $\cdot$ Fexapotide triflutate $\cdot$ Urology $\cdot$ Focal therapy

\section{Introduction}

There is an unmet need for treatments for low-grade prostate cancer $(\mathrm{PCa})$ that produce minimal collateral tissue damage and unintended sexual, urinary, and bowel function side

Neal Shore

NShore@gsuro.com

1 Carolina Urologic Research Center, Myrtle Beach, SC, USA

2 Icahn School of Medicine at Mount Sinai, New York, NY, USA

3 Chesapeake Urology Research Associates, Baltimore, MD, USA

4 Chesapeake Urology Research Associates, Towson, MD, USA

5 First Urology, Louisville, KY, USA effects [1-23]. Grade groups were first proposed by authors at Johns Hopkins Hospital led by Dr. Epstein [21], validated in a large multi-institutional study [22], and subsequently endorsed by the 2014 International Society of Urological Pathology Consensus Conference [23] and the WHO.

\footnotetext{
6 Willamette Urology, Salem, OR, USA

7 Urological Associates of Southern Arizona, Tucson, AZ, USA

8 San Diego Clinical Trials, San Diego, CA, USA

9 Freedman Urology, Las Vegas, NV, USA

10 UT Southwestern Department of Urology, Lewisville, TX, USA

11 Urology Group of New Mexico, Albuquerque, NM, USA

12 Johns Hopkins Medical Institutions, Baltimore, MD, USA
} 
Gleason Grade Group 1 is the most commonly diagnosed $\mathrm{PCa}$ and is considered very low-to-low risk by the National Comprehensive Cancer Network (NCCN) criteria. However, these cancers, albeit low risk, are still infrequently capable of biologic progression, and thus cause ongoing patient anxiety. Often, these low-risk cancer patients may still receive interventional therapies which can result in urinary, bowel, and sexual side effects. The natural history of these indolent, well-differentiated prostate cancers, and their management can be influenced by age, performance status, co-morbidities, sociodemographic factors, and genomic factors [24-36]. An overarching difficulty in the management of low-risk prostate cancer patients is that validations with long-term prospective outcomes are essentially prohibitively delayed due to the 15-20 years required for mortality data and the unrealistic likelihood of adequate recruitment. Most authorities, therefore, agree that more pragmatic parameters of objective clinical and pathological progression are currently the most realistic approach to assessment of efficacy and safety [1-20].

Fexapotide triflutate (FT) is a new molecular entity with pro-apoptotic effects, delivered by intraprostatic injection, which has been investigated for the treatment of both lower urinary tract symptoms due to BPH and for low-grade localized PCa. FT has been administered in prospective randomized placebo controlled double-blinded long-term BPH trials involving over 1200 men, and shown to be well tolerated and to provide long-term BPH efficacy without significant adverse effects [37-40]. This report presents 4-year data from a 7-year study of 146 patients with Grade Group 1 (Gleason 6) T1c PCa, randomized to treatment with Fexapotide Triflutate (FT) or active surveillance (AS), and which included an elective crossover (CO) group from AS to active drug. To our knowledge, this is the first long-term prospective randomized-controlled study of an intraprostatic molecular injectable treatment for low-grade localized PCa to be reported.

\section{Patients and methods}

Study NX03-0040 was a Phase 2 multi-center prospective open label two-dose $(2.5 \mathrm{mg}$ and $15 \mathrm{mg}$ ) level clinical safety and efficacy evaluation of FT injection for the treatment of lowrisk, localized (T1c) PCa, comparing FT 2.5-mg and 15-mg single dose to AS, and including an elective AS CO group. The study was conducted at 28 U.S. urological investigational sites (44 sites approved and initiated; 30 with patient screening; 28 with patient enrollments) from 2012 to 2018, with protocols approved by institutional review boards (clinicaltrials. gov identifier NCT01620515). Informed consent was obtained from all individual participants included in the study. Patients were enrolled based on the following criteria: diagnosis of T1c
PCa Grade Group 1; prostate biopsy within previous 6 months ( $\geq 10$ cores; single core positive; $\leq 50 \%$ in the single positive core); PSA $\leq 10 \mathrm{ng} / \mathrm{mL}$; and no previous treatments for prostate cancer. Patients' assessments additionally included International Prostate Symptom Score (IPSS) at baseline and at follow-up. Patients were centrally randomized in a $1: 1: 1$ ratio of FT 2.5-mg:FT 15-mg:AS; by a computer-generated randomization schedule executed by non-study personnel at an independent randomization service provider with no contact except by interactive voice response system. Patients and investigational staff were blinded as to which FT dosages (FT $15 \mathrm{mg}$ vs $2.5 \mathrm{mg}$ ) were administered in the treatment groups. AS patients were not given sham treatments. 267 patients were screened, with 146 patients enrolled, and 141 patients qualified for efficacy analysis (Intent-to-Treat). Three groups were initially randomized (Study Population, Table 1): FT $15 \mathrm{mg}$ ( $n=48$ randomized, $n=47$ injected), FT $2.5 \mathrm{mg}(n=49$ randomized, $n=48$ injected) by transrectal intraprostatic injection; and AS ( $n=49)$, and two smaller elective groups of AS to FT COs after the 6-week post-randomization biopsy (FT $2.5 \mathrm{mg}$ $n=10$; FT $15 \mathrm{mg} n=8$ ). CO patients were required to have completed the initial 6 weeks per protocol and to continue to fulfill inclusion/exclusion criteria at the time of CO. Seven initial visits included: Visit 1: screening; Visit 2 (Day 1): dosing; Visit 3 (Day 2); Visit 4 (Day 4); Visit 5 (Day 10); Visit 6 (Day 45): biopsy; and Visit 7 (Day 60); biopsy follow-up. Follow-up Visits (up to 5 years): PSA (every 6 months); physical examination (every 12 months); prostate biopsies (every 18 months). Blinded prostate biopsies at screening and at 45 days were read by Dr. J. Epstein, Johns Hopkins Medical Institutions. Long-term biopsies after 45 days were reported by the local pathology services in conjunction with the individual investigational sites. Interim safety analyses by an Independent Safety Data Monitoring Committee (IDMC) were scheduled (i) after the first ten patients dosed with FT (both dosage groups combined $n=10$ ), (ii) after the first ten FT 15-mg dose group, and (iii) after $n=50$ both groups FT combined. If there was excessive drug-related toxicity, the IDMC would stop the trial. The injection procedure for FT intraprostatic treatment has been described in detail previously [37-40] and was directed to the same quadrant as the cancer on initial biopsy. FT is supplied as a sterile lyophilized powder that is reconstituted in $10-\mathrm{mL}$ sterile phosphate buffered saline and injected into prostate by the transrectal route under transrectal ultrasound (TRUS) guidance by standard technique using a conventional \#22 gauge sterile needle.

\section{Statistical methods}

\section{Statistical analysis plan}

Primary endpoint was presence or absence of cancer in initially positive baseline quadrant focus (BLF) based on 
Table 1 Study population

\begin{tabular}{llll}
\hline Group & FT 2.5 mg & FT 15 mg & AS $^{\mathrm{a}}$ \\
\hline$N$ randomized & 49 & 48 & 49 \\
Age (mean in years) (SD) & $64.0(7.1)$ & $64.4(7.7)$ & $62.6(7.0)$ \\
Race & & & \\
$\quad$ Caucasian & 47 & 45 & 43 \\
African-American & 2 & 2 & 4 \\
$\quad$ Other & 0 & $1^{\mathrm{b}}$ & $2^{\mathrm{c}}$ \\
PSA (mean) (SD) & $4.7(2.3)$ & $4.2(1.9)$ & $4.3(1.9)$ \\
IPSS (mean) (SD) & $9.6(6.4)$ & $8.3(5.8)$ & $10.9(8.8)$ \\
Prostate volume (mean) (SD) & $52.3(23.1)$ & $46.1(15.9)$ & $49.7(24.7)$ \\
Clinical stage t(1c) t(2a) & $49 / 49$ & $48 / 48$ & $49 / 49$ \\
Biopsy (ISUP Grade 1) & $49 / 49$ & $48 / 48$ & $49 / 49$ \\
Gleason 3+3 number of positive cores & & & $49 / 49$ \\
1 & $49 / 49$ & $48 / 48$ & $10.4 \%(11.5 \%)$ \\
Mean lesion \% of positive core (SD) & $9.5 \%(8.8 \%)$ & $12.3 \%(12.2 \%)$ & \\
\hline
\end{tabular}

${ }^{\mathrm{a}} \mathrm{CO}$ patients are included in $\mathrm{AS}$ at baseline as randomized. $\mathrm{CO}$ patients are included in their respective FT groups for outcome listings in Table 3

${ }^{\mathrm{b}}$ Caucasian-American Indian

${ }^{\mathrm{c}}$ American-Indian; Asian

blinded assessments of prostate biopsies and PSA. Secondary endpoints included: change in median tumor grade in each treatment group in (i) BLF, and (ii) in the entire prostate; and change in mean tumor volume in each treatment group (estimated by tumor \% in biopsy section) in (iii) BLF, and (iv) in the entire prostate. Cancer progression was assessed by clinical evaluation, long-term serial biopsies (every 18 months or earlier, if for cause), PSA (every 6 months or earlier), incidences of surgery or radiotherapy for PCa with or without histological upgrades, and surgical pathology results. All patients with clinical and/or pathological progression (increased Gleason grade $>3+3$ in their prostate overall, or post-randomization treatment with prostatectomy or radiotherapy and/or chemotherapy) were included in the statistical analysis regardless of when they exited the study. CO subjects were included in the AS group for the initial 6-week protocol measures only. After CO, they were included in the FT group of their respective FT dosage received. Subjects lost to followup or who dropped out, without clinical or pathological progression, were included in pathological analysis at 18 , 36 , and 48 months if there was $\geq 18, \geq 36$, or $\geq 48$-month biopsy data, respectively, but were excluded if they were lost prior to 18-month biopsy, and were excluded from later calculations if subsequently lost or withdrawn. Corrections for multiple comparisons were not done for the significance values which are reported separately. For BLF in patients with surgery or radiation and no biopsy, baseline was carried forward. Statistical assistance was provided by Sherryl Baker PhD (Everest Clinical Research, Little Falls NJ).

\section{Results}

Patient disposition is summarized in CONSORT diagram (Fig. 1). First patient enrollment was June 6, 2012, and last patient enrollment $(n=146)$ was Feb 11, 2014. Long-term follow-up data were analyzed as of 78-month post-first patient enrollment (which was 5-year post-last patient enrollment). Mean and (median) ages at randomization were AS 63.2 (64), FT $2.5 \mathrm{mg} 63.7$ (63), and FT $15 \mathrm{mg} 63.9$ (65) years. There were two $(1.37 \%)$ patients who dropped out before (day 1) study treatment and two (1.37\%) dropped out (without cause) before the first post-randomization biopsy at 45 days. Prior to the 18 -month biopsy assessment $n=14$ (9.6\%) of subjects had exited the study due to pathological progression of their PCa and $n=11(7.5 \%)$ exited without progression due to the decision to receive interventions such as surgery or radiotherapy for their PCa, with 5 (3.4\%) patients lost to follow-up (LTFU) and 32 (21\%) withdrew consent without documented progression of their PCa.

\section{Safety results}

Consistent with previous U.S. clinical trial data $(n>1200)$ for FT $2.5 \mathrm{mg}$, the vast majority of drug injection-related adverse events (AEs) included injection procedure-related AEs (e.g., transient hematuria in $6.1 \%$; transient dysuria in $1.7 \%$; transient hematospermia in $2.6 \%$ ) antibiotic prophylaxis-related AEs (e.g., transient diarrhea in $26.1 \%$; transient nausea in 8.7\%; transient constipation in $1.7 \%$ ) (Table 2), and resolved uneventfully. There were no FT drug-related AEs. This is consistent with clinical pharmacokinetic (PK) data which 


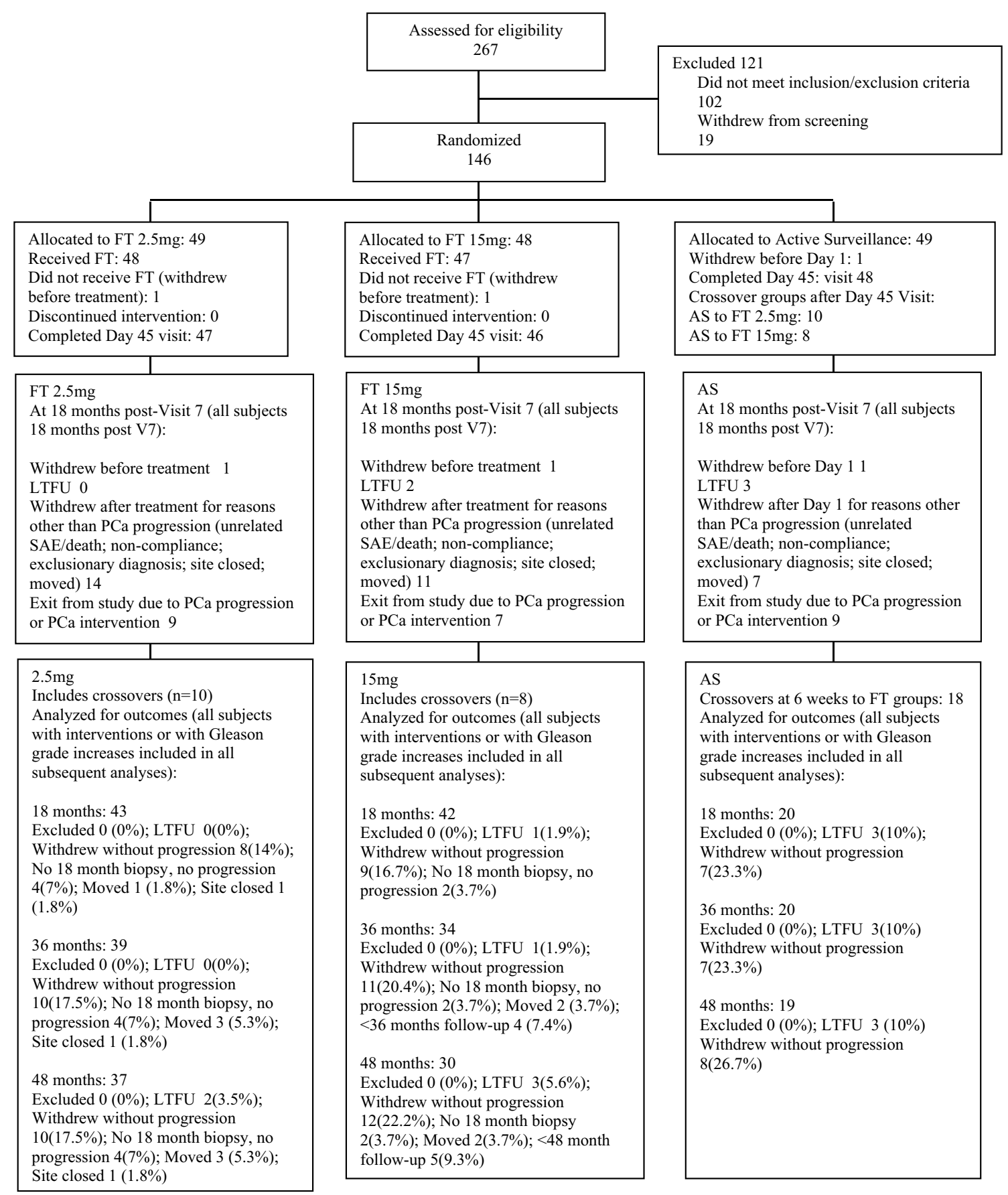

Fig. 1 Consort diagram

have shown no detectable levels in plasma at 1-, 5-, 10-, and 20-min post-injection, and with clinical immunological data showing no antibody response to FT after injection and after re-injection [37-40]. In the present study, total testosterone levels in FT groups were not reduced compared to controls at
45 days. There were no long-term clinically significant lower urinary tract side effects in any subjects. Lower urinary tract symptoms (LUTS) changes as measured by IPSS after 6 weeks showed no difference between pooled FT subjects and AS [mean change from baseline all FT-treated subjects - 1.05 (SD 3.83) vs AS 0.02 (3.41), NS, $t$ test]. 
Table 2 Treatment-related adverse events (AEs) in the first year after treatment

\begin{tabular}{|c|c|c|c|c|}
\hline $\mathrm{AE}$ & $\begin{array}{l}\text { Combined FT } \\
(n=115) \text { group } \\
\text { AEs: } n(\%)\end{array}$ & $\begin{array}{l}\text { FT } 2.5 \mathrm{mg}(n=59) \\
\text { group } \\
\text { AEs: } n(\%)\end{array}$ & $\begin{array}{l}\text { FT } 15 \text { mg }(n=56) \\
\text { group } \\
\text { AEs: } n(\%)\end{array}$ & $\begin{array}{l}\mathrm{AS}^{\mathrm{a}} \\
(n=49) \\
\text { group } \\
\text { AEs: } n \\
(\%)\end{array}$ \\
\hline \multicolumn{5}{|l|}{ Procedure-related ${ }^{\mathrm{b}}$} \\
\hline Dysuria & $2(1.7)$ & $1(1.7)$ & $1(1.8)$ & 0 \\
\hline Haematochezia & $3(2.6)$ & 0 & $3(5.4)$ & 0 \\
\hline Haematospermia & $3(2.6)$ & $1(1.7)$ & $2(3.6)$ & 0 \\
\hline Haematuria & $7(6.1)$ & $3(5.1)$ & $4(7.1)$ & 0 \\
\hline Penile pain & $2(1.7)$ & 0 & $2(3.6)$ & 0 \\
\hline Rectal pain & 0 & 0 & 0 & $1(2.0)$ \\
\hline \multicolumn{5}{|l|}{ Antibiotic-related $^{\mathrm{b}}$} \\
\hline Arthralgia & $2(1.7)$ & $2(3.4)$ & 0 & 0 \\
\hline Constipation & $2(1.7)$ & 0 & $2(3.6)$ & 0 \\
\hline Diarrhoea & $30(26.1)$ & $19(32.2)$ & $11(19.6)$ & $1(2.0)$ \\
\hline Dysgeusia & $3(2.6)$ & 0 & $3(5.4)$ & 0 \\
\hline Headache & $3(2.6)$ & $3(5.1)$ & 0 & 0 \\
\hline Nausea & $10(8.7)$ & $6(10.2)$ & $4(7.1)$ & 0 \\
\hline
\end{tabular}

All FT-related AEs with combined FT groups $n>1$, and all related AS AEs $\geq 1 \%$. Excludes biopsy-related AEs

There were no FT drug-related AEs. There were no serious AEs (SAEs)

${ }^{a}$ Includes all randomized AS subjects. Crossover patient AEs included with AS group prior to crossover. Post-crossover AEs included in FT groups

${ }^{\mathrm{b}}$ Self-limited and brief duration ( $n=41 / 67 \leq 4$ days $)$

\section{Efficacy results (Table 3; Fig. 2): all comparisons unless otherwise listed are by Pearson exact chi-square}

At 18-month biopsies, primary endpoint was met for FT

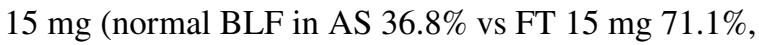
$\mathrm{p}=0.0214$; pooled FT $65.8 \%$, NS; FT $2.5 \mathrm{mg} 61 \%$, NS). Secondary endpoints were as follows: (1) Median Gleason grade in each treatment group in BLF at 18 months was AS $3+3$ (Group Grade 2 or higher in 4/19), vs benign for each of FT $15 \mathrm{mg}$ (Group Grade 2 or higher in 1/38) ( $p=0.0066$ ), FT $2.5 \mathrm{mg}$ (Group Grade 2 or higher in 2/41) $(p=0.0593)$, and pooled FT groups (Group Grade 2 or higher in 3/79) $(p=0.0109)$. (2) Median Gleason grade in each treatment group for entire prostate at 18 months was benign for FT $15 \mathrm{mg}$ (Group Grade 2 or higher in 3/34) $(p=0.0044)$ and benign for pooled FT (Group Grade 2 or higher in 9/70) $(p=0.0086)$ vs $3+3$ for AS (Group Grade 2 or higher in 7/17), and $3+3$ for FT $2.5 \mathrm{mg}$ (Group Grade 2 or higher in 6/36). (3) BLF mean tumor volume in each treatment group (estimated by biopsy \%) at 18 months was pooled FT group (-58.5\%) vs AS controls $(+68.8 \%$, $p=0.0189, t$ test $)($ FT $15 \mathrm{mg},-59 \%$, FT $2.5 \mathrm{mg},-$
58\%). (4) Entire prostate mean tumor volume change at 18 months was significantly lower in pooled FT group $(+41.5 \%)$ compared to AS controls $(+279.7 \%, p=0.0134)$ (FT $15 \mathrm{mg}+32.7 \%$, FT $2.5 \mathrm{mg}+47.8 \%, t$ tests).

Data analysis of pathological progression (Table 3; Fig. 2a, b) was done with the exclusion of negative (ie nonprogression) patients where there was no biopsy or surgical pathology data from the relevant time point or later. The pathological results for long-term Gleason grade (in addition to the median tumor grade progression differences above) included (all comparisons below by Pearson exact chi-square):

1. Percentage of subjects with increase of Gleason score to Grade Group 2 (Gleason score $3+4=7$ ) or higher on follow-up biopsy or RP surgical pathology in prostate overall was significantly reduced in FT vs AS [18 months: AS 41.2\% vs FT $15 \mathrm{mg} 8.8 \%$ (- 78.6\%, $p=0.0102)$; FT $2.5 \mathrm{mg} 16.7 \%(-59.5 \%, p=0.0858)]$; pooled FT $12.9 \%(-68.7 \%, p=0.0129)$; 36 months: AS $56.3 \%$ vs FT $15 \mathrm{mg} 18.2 \%(-67.7 \%, p=0.0199)$; FT $2.5 \mathrm{mg} 26.9 \%$, (- 52.2\% NS); pooled FT $22.9 \%$ ($59.3 \%, p=0.0265)$; 48 months: AS $71.4 \%$ vs FT $15 \mathrm{mg}$ $33.3 \%(-53.4 \%, p=0.0656)$; FT $2.5 \mathrm{mg} 62.5 \%$, NS; 
Table 3 Progression outcomes

\begin{tabular}{|c|c|c|c|c|}
\hline & FT $2.5 \mathrm{mg}$ & FT $15 \mathrm{mg}$ & Pooled FT & AS \\
\hline \multicolumn{5}{|c|}{$\begin{array}{l}\text { (a) } \% \text { (Proportion) with Gleason } \geq 3+4 \text {, biopsies and RP surgical } \\
\text { pathology }\end{array}$} \\
\hline 18 months & $16.7(6 / 36)$ & $8.8(3 / 34)^{1}$ & $12.9(9 / 70)^{2}$ & $41.2(7 / 17)$ \\
\hline 36 months & $26.9(7 / 26)$ & $18.2(4 / 22)^{3}$ & $22.9(11 / 48)^{4}$ & $56.3(9 / 16)$ \\
\hline 48 months & $\begin{array}{l}62.5 \\
(10 / 16)\end{array}$ & $33.3(5 / 15)$ & $48.4(15 / 31)$ & $71.4(10 / 14)$ \\
\hline \multicolumn{5}{|c|}{$\begin{array}{l}\text { (b) } \% \text { (Proportion) with both Gleason } \geq 3+4 \text { biopsies and RP surgi- } \\
\text { cal pathology, and interventions }{ }^{\text {a }}\end{array}$} \\
\hline 18 months & $9.8(4 / 41)$ & $7.7(3 / 39)$ & $8.8(7 / 80)$ & $36.8(7 / 19)$ \\
\hline 36 months & $16.7(5 / 30)$ & $14.3(4 / 28)$ & $15.5(9 / 58)$ & $47.1(8 / 17)$ \\
\hline 48 months & $30.4(7 / 23)$ & $16.7(4 / 24)^{5}$ & $23.4(11 / 47)^{6}$ & $62.5(10 / 16)$ \\
\hline \multicolumn{5}{|c|}{$\begin{array}{l}\text { (c) } \% \text { (Proportion) with Gleason } \geq 3+4 \text { biopsies and RP surgical } \\
\text { pathology }{ }^{b}\end{array}$} \\
\hline 18 months & $12.8(6 / 47)$ & $6.5(3 / 46)^{7}$ & $9.7(9 / 93)^{8}$ & $25.9(7 / 27)$ \\
\hline 36 months & $18.9(7 / 37)$ & $11.8(4 / 34)^{9}$ & $\begin{array}{l}15.5 \\
(11 / 71)^{10}\end{array}$ & $34.6(9 / 26)$ \\
\hline 48 months & $37(10 / 27)$ & $18.5(5 / 27)$ & $27.8(15 / 54)$ & $41.7(10 / 24)$ \\
\hline \multicolumn{5}{|c|}{$\begin{array}{l}\text { (d) } \% \text { (Proportion) with both Gleason } \geq 3+4 \text { biopsies and RP surgi- } \\
\text { cal pathology, and interventions } \mathrm{s}^{\mathrm{a}, \mathrm{b}}\end{array}$} \\
\hline 18 months & $7.7(4 / 52)^{11}$ & $5.9(3 / 51)^{12}$ & $6.8(7 / 103)^{13}$ & $24.1(7 / 29)$ \\
\hline 36 months & $12.2(5 / 41)$ & $10(4 / 40)^{14}$ & $11.1(9 / 81)^{15}$ & $29.6(8 / 27)$ \\
\hline 48 months & $20.6(7 / 34)$ & $\begin{array}{l}11.1 \\
(4 / 36)^{16}\end{array}$ & $\begin{array}{l}15.7 \\
(11 / 70)^{17}\end{array}$ & $38.5(10 / 26)$ \\
\hline
\end{tabular}

${ }^{a} \mathrm{RP}$, radiotherapy, or chemotherapy

${ }^{b}$ Including all patients with $\geq 2$ biopsies

${ }^{1} p=0.0102 ; \quad{ }^{2} p=0.0129, \quad{ }^{3} p=0.0199, \quad{ }^{4} p=0.0265, \quad{ }^{5} p=0.0059$, ${ }^{6} p=0.0064$ (1-6 Pearson exact chi-square), ${ }^{7} p=0.0199,{ }^{8} p=0.0288$, ${ }^{9} p=0.033,{ }^{10} p=0.0392,{ }^{11} p=0.0383,{ }^{12} p=0.0176,{ }^{13} p=0.0074$, ${ }^{14} p=0.0398,{ }^{15} p=0.0221,{ }^{16} p=0.011,{ }^{17} p=0.0166$ (7-17 Pearson chi-square)

pooled FT $48.4 \%(-32.2 \%$, NS). Median tumor grades at 36 months were Gleason $3+4$ for AS vs Gleason $3+3$ for each of FT $15 \mathrm{mg}$, FT $2.5 \mathrm{mg}$, pooled FT; and those at 48 months were Gleason $3+4$ for AS vs $3+3$ for FT $15 \mathrm{mg}$, pooled FT; and $3+4$ for FT $2.5 \mathrm{mg}$.

2. Patients treated with FT $15 \mathrm{mg}$ and pooled FT group had significantly less increase of treatment side Gleason score to Grade Group 2 (Gleason score $3+4=7$ ) or higher at 18 and 36 months [18 months: AS $33.3 \%$ vs FT $15 \mathrm{mg} 8.8 \%(-73.6 \%, p=0.0466)$; FT $2.5 \mathrm{mg}$ $11.8 \%(-64.6 \%, \mathrm{NS})]$; pooled FT $10.3 \%(-69.1 \%$, $p=0.0365) ; 36$ months: AS 50\% vs FT $15 \mathrm{mg} 18.2 \%$ $(-63.6 \%, p=0.0665)$; FT $2.5 \mathrm{mg} 20.8 \%(-58.4 \%, \mathrm{NS})$; pooled FT $19.6 \%(-60.8 \%, p=0.0379)$.

3. Percentage of subjects with primary Gleason pattern $(\geq 4)$ on follow-up biopsies and RP surgical pathology in prostate overall was reduced in pooled FT groups vs AS [18 months: AS $17.6 \%$ vs pooled FT $3.1 \%$ (-82.4\%, $p=0.0597)$ ]; 36 months: AS $26.7 \%$ vs pooled FT $5.1 \%$ $(-80.9 \%, p=0.0439)$. The percentage of AS subjects with biopsy primary pattern $\geq 4$ at 3 years was $15.4 \%$, compared to FT $2.5 \mathrm{mg} \mathrm{5 \% ,} \mathrm{FT} 15 \mathrm{mg} 5.2 \%$, and pooled FT $5.1 \%$.

Prospective assessment of $\mathrm{PCa}$ progression showed evidence of drug benefit. After 4 years, the percentage of control AS patients who received surgery or radiotherapy for their PCa was $68.4 \%$, vs FT $15 \mathrm{mg} \mathrm{31 \%} \mathrm{(-} \mathrm{54.7 \% ,}$ $p=0.0177)$, and vs combined FT groups $40 \%(-41.5 \%$, $p=0.0374)$. The percentage of control AS patients with surgery or radiotherapy with Gleason grade progression from baseline in the entire gland was $62.5 \%$, vs FT $15 \mathrm{mg}$ $16.7 \%(-73.3 \%, p=0.0059)$, vs FT $2.5 \mathrm{mg} 30.4 \%(-51.4 \%$, $p=0.0586)$, and vs pooled FT dosages $23.4 \%(-62.6 \%$, $p=0.0064$ ) (Fig. 2b, d). Total $n$ of CO patients with pathological data $\geq 36$ months: six (FT $15 \mathrm{mg} / 2.5 \mathrm{mg}: 3$ patients each) and $\geq 48$ months: two (FT $15 \mathrm{mg} / 2.5 \mathrm{mg}$ : 1 patient each); calculations without $\mathrm{CO}$ subjects had no significant effect on the above progression results. Two patients (both FT $2.5 \mathrm{mg}$ ), one with Gleason grade $5+3$ (LTFU) and a second with Gleason $3+4$ (site closed), were included in the pathological results, but were excluded from intervention results, because there was no confirmation of treatment despite efforts to follow-up. Two patients (FT $15 \mathrm{mg}$ ), one with Gleason grade $4+4$, and a second with $4+3$ (age 88 ), and one patient (FT $2.5 \mathrm{mg}$ ) with Gleason $3+4$, were untreated after 5 years of surveillance, and were included in all results. Gleason upgrades overall were $67.9 \%$ in BLF or immediately adjacent quadrants, $32.1 \%$ were not adjacent, with $24.9 \%$ contralateral. Although the incidence of progression was significantly higher in untreated AS subjects, there were no significant differences between FT and AS groups in the above quadrant localization relative proportions. Consistency of quadrant localization reporting in SP specimens after study exit treatments had limitations due to non-uniform external processing and non-central reading after the 45-day biopsy.

Post hoc analysis of baseline PSA density showed a minority of patients (15.8\%) had PSA density $\geq 0.15 \mathrm{ng} / \mathrm{mL} /$ $\mathrm{cc}^{3}$. There were no significant differences between treatment randomization groups re baseline proportions of higher PSA density patients, nor in proportions of patients with postrandomization Gleason grade increases in higher vs lower baseline PSA density patients. Post hoc sensitivity testing of Gleason grade progression was done by including all randomized subjects in all groups with $\geq 2$ biopsies, which showed AS progression values of $25.9 \%$ and $34.6 \%$ at 18 and 36 months, compared to FT $15 \mathrm{mg} \mathrm{6.5 \%}(p=0.0199)$ and $11.8 \%(p=0.033)$, and compared to pooled FT $9.7 \%$ $(p=0.0288)$ and $15.5 \%(p=0.0392)$ (Table 3; Fig. 2c, d).

Dose-response long-term data showed FT 15-mg superiority to FT $2.5 \mathrm{mg}$ in most endpoints and overall cancer progression. (1) Four-year incidence of surgery and 


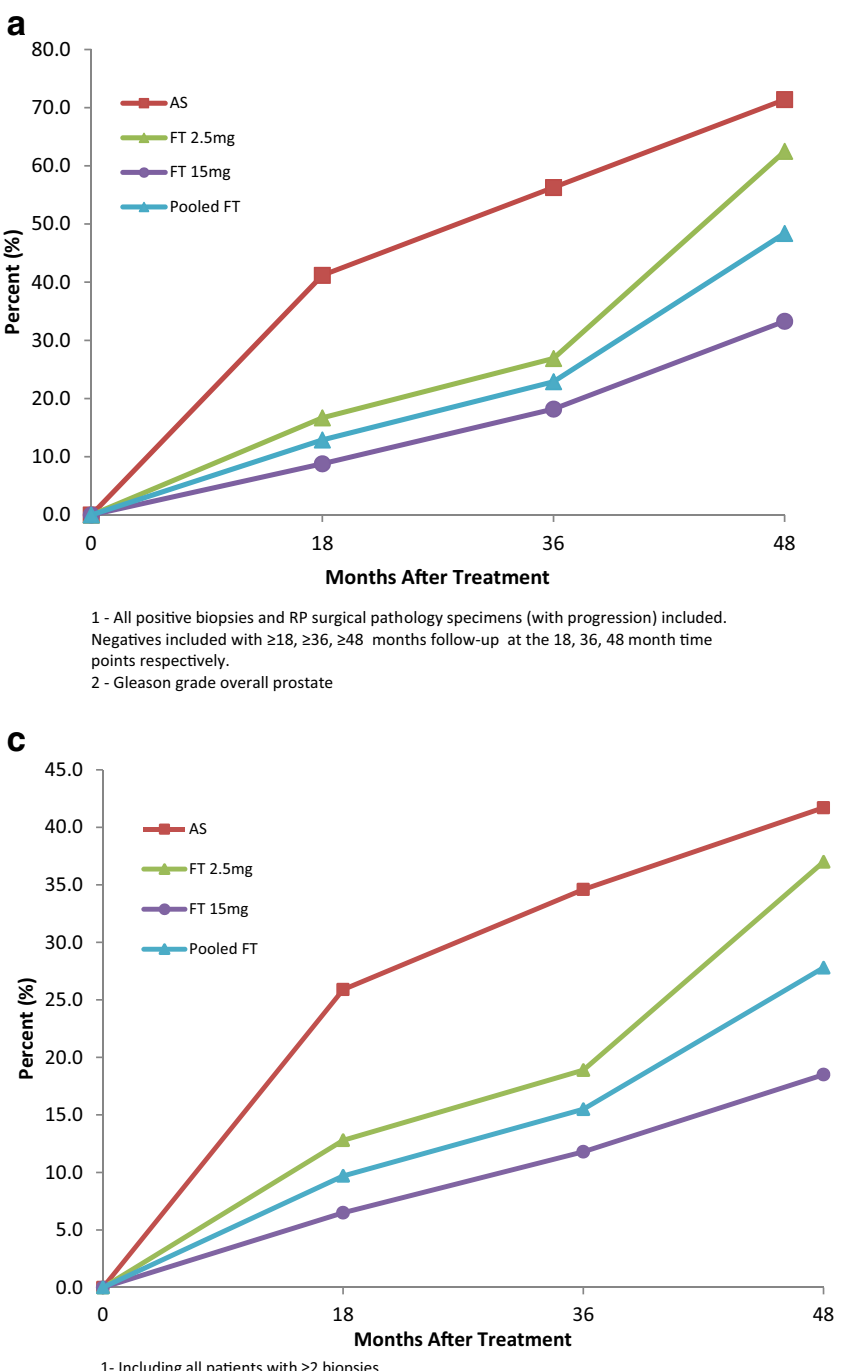

1- Including all patients with $\geq 2$ biopsies

Fig. 2 Outcomes of clinical and pathological progression vs time in FT-treated and AS control patients. a Cumulative percentage of patients with increased Gleason grade biopsies and/or RP surgical pathology specimens vs time after treatment. FT $15 \mathrm{mg}$ and pooled FT groups statistically significant reduction vs AS control (in Table 3). b Cumulative percentage of patients with interventions (surgery and radiotherapy) with Gleason grade biopsies and/or

radiotherapy for all causes was significantly reduced in the FT $15 \mathrm{mg}$ group (31\%), compared to control AS (68.4\%, $p=0.0177)$, while the $2.5-\mathrm{mg}$ group change $(48.4 \%)$ was not statistically significant. (2) 3-year incidence of Gleason grade increase in prostate overall was significantly reduced $(-67.7 \%)$ in patients who received FT $15 \mathrm{mg}(18.2 \%)$ compared to AS control group $(56.3 \%, p=0.0199)$ but in the FT $2.5 \mathrm{mg}$ group $(26.9 \%, p=0.1009)$ was not. (3) Incidence of 18 -month Gleason grade increase was significantly less in the FT $15-\mathrm{mg}$ group compared to AS in the prostate overall $(-78.6 \%, p=0.0102)$ and in the treated prostate lobe $(-73.6 \%, p=0.0466)$, whereas the reductions in the $2.5-\mathrm{mg}$
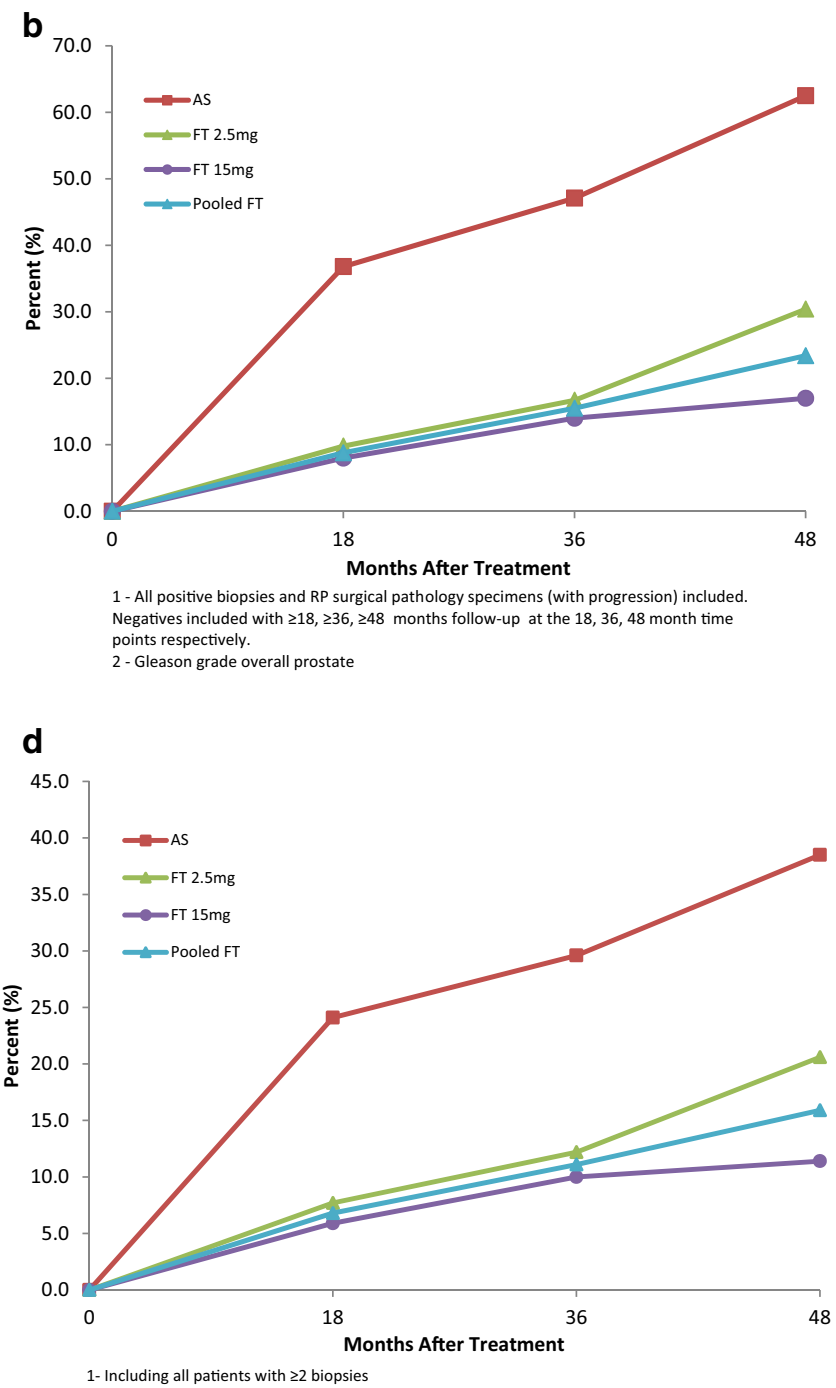

RP surgical pathology specimens vs time after treatment. FT $15 \mathrm{mg}$ and pooled FT groups' statistically significant reduction vs SAS control (in Table 3). c Cumulative percentage of patients with increased Gleason grade vs time after treatment (as in a, also including all subjects with $\geq 2$ biopsies). d Cumulative percentage of patients with increased Gleason grade and intervention vs time after treatment (as in $b$, also including all subjects with $\geq 2$ biopsies).

group did not reach statistical significance (- 59.5\%, NS and $-64.6 \%$, NS, respectively).

PSA mean change baseline to post-randomization nadir was significantly reduced in FT-treated groups but not in AS controls at 18 months (FT $2.5 \mathrm{mg}-16.7 \%, p=0.0011$; FT $15 \mathrm{mg}-18.6 \%, p=0.0015)$; pooled FT groups $-17 \%$, $p<0.0001$ vs AS $(-13.3 \%$, NS) and at 4 years (FT $2.5 \mathrm{mg}$ $-21.4 \%, p=0.0004$; FT $15 \mathrm{mg}-20.4 \%, p=0.0065$ ); pooled FT groups $-20.9 \%, p<0.0001$; AS $(-12.5 \%$, NS) (one-sample $t$ tests).

With terminal deoxynucleotidyl transferase deoxyuridine triphosphate nick end (TUNEL) staining of biopsies, foci of 
glandular cells undergoing apoptotic cell death were identified in FT-treated subjects in their 45-day biopsies (Fig. 3). There was no TUNEL positivity identified in control AS biopsies. These histochemical results will be expanded in a separate research report.

\section{Discussion}

The results of this long-term prospective study show that after a single targeted FT injection, there is evidence of statistically significant long-term inhibition of PCa progression both clinically and histologically. Clinical progression after 4 years was reduced by FT whether calculated by occurrence of interventions for $\mathrm{PCa}$ with increased Gleason grade in overall prostate ( $-73.3 \%$ for FT 15 -mg group compared to AS, $-62.6 \%$ for pooled FT groups); or by interventions with or without Gleason increase (-54.7\% for FT $15-\mathrm{mg}$ group compared to AS; $-41.5 \%$ for pooled FT groups). Pathological progression after 3 years was diminished as determined by comparison of prostate overall incidence of increased Gleason grade ( $-67.7 \%$ incidence of Gleason grade increase for FT 15-mg group compared to AS group, $-59.3 \%$ for pooled FT groups); and 3-year comparison of treatment side hemi-prostate $(-63.6 \%$ incidence of Gleason grade increase on side of FT 15 -mg treatment compared to AS, $-60.8 \%$ for pooled FT groups). Three-year incidence of primary pattern $\geq 4$ was reduced $-80.9 \%$ in pooled FT patients compared to AS arm.

It should be emphasized that this study prospectively compared single injection of two different single doses of FT to an AS cohort. Better results may, therefore, be potentially possible after repeated injection(s). In BPH trials for FT, repeat injection has produced greater long-term improvement in BPH parameters compared to single-dose treatment, without any additional safety risk [37, 38, 41-43].
The injections in this study were targeted by routine ultrasound to the quadrant where the qualifying biopsy had identified the baseline T1c PCa single lesion. Although FT is not measurable (not present) by pharmacokinetic sampling outside of prostate at any time point after injection [37-40], the injection of 10-mL FT solution can be visualized by ultrasound to diffuse within the hemi-prostate well beyond the $10-\mathrm{mL}$ volume space [41-43]. Statistically significant reduced PCa progression within the treatment side hemi-prostate was unexpected, but can probably in part be explained by the visualized diffusion of FT within the ipsilateral lobe and contact with other foci of undetected tumor at the time of treatment. Enhanced imaging is expected to improve targeting accuracy compared to TRUS alone. However for the reasons cited above, the 10-mL injection with visualized diffusion by TRUS was likely to have reached much more of the prostate lobe than only the targeted lesion.

It is unknown at this time if the beneficial effect in outcomes with FT in this study is due to destruction of primary grade 6 foci some of which would have progressed to higher grade, or due to destruction of pre-existent undetected higher grade foci different from the baseline focus identified, or to missed higher grade foci within the initial focus, or whether the decrease in progression compared to AS is through another hypothetical mechanism such as inhibition of other premalignant cellular targets, or combinations of two or more of the above. Discussion of these currently unverifiable mechanisms is beyond the scope of this report. The higher Gleason grade foci found in the patients with progression were $67.9 \%$ in BLF or adjacent quadrants, and only $24.9 \%$ were contralateral, which suggests that most progression was emanating (whether de novo or pre-existent) from the baseline identified foci or closely adjacent tissue.

The main potential roles of a validated non-chemotherapeutic non-toxic molecular approach to low-grade $\mathrm{PCa}$ are (1) the possibility to delay treatment interventions which may be associated with undesirable secondary effects, and
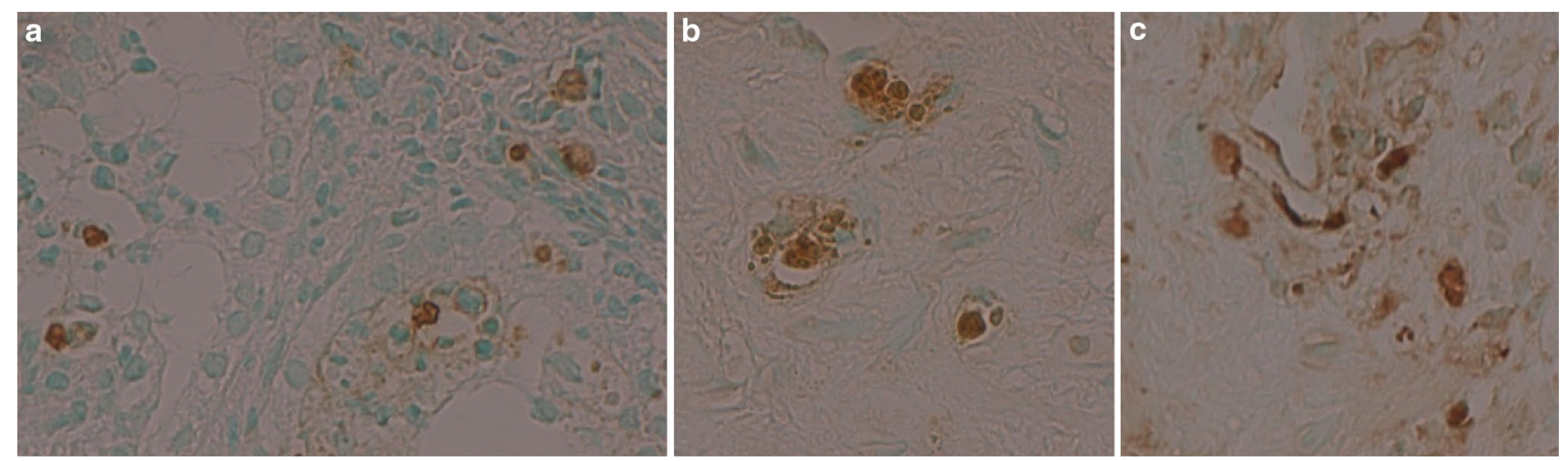

Fig. 3 a-c Immunohistochemical TUNEL staining of 6-week biopsies from FT-treated patients showing TUNEL positivity (dark brown) indicating apoptosis of prostate glandular cells after FT injection into prostate. $\times 400$ (figure courtesy of Nymox Corp) 
(2) the option to continue an ongoing AS strategy yet offer patients an opportunity for potential cancer ablation. For many patients, this adjunct to surveillance might be helpful for the persistent and considerable uncertainties, anxieties, and psychological/emotional burdens, which may negatively impact quality of life when only selecting AS.

There are limitations to this long-term study: (1) single dosing was utilized and the additional potential benefits of multiple dosing were not explored in cohorts, (2) potential combination(s) with other non-interventional modalities (e.g., molecular) was not addressed, (3) patients with more extensive low-grade PCa were not in the trial, so the benefit for these important groups remains unknown (e.g., Gleason 6 with biopsy proven multifocal disease, Gleason 6 with lesion(s) $>50 \%$ of biopsy core, Gleason $3+4$, and others), (4) prospective sub-groups were not explored (e.g., ethnic; and phenotypic sub-groups), (5) MRI, which would help to define intervention sites, was not a required part of the 2012 protocol for this study, and (6) blinded central review was done on all first-year biopsies $(n=287)$, but subsequent biopsies and surgical pathology from RP specimens were done at the local institutions where standardization was less uniform and inter-observer variability was a potential source of imprecision. All of these additional aspects, and perhaps, other considerations remain to be addressed in further studies.

This study was a prospective randomized multi-center parallel group controlled study designed to test safety and effectiveness of FT treatment and AS compared to AS alone, in a group of 147 men with recent biopsy Grade Group 1 PCa. Larger single-center studies of AS cohorts have reported histological progression rates in the $12-51 \%$ range after median times of 1.5-6.4 years, most commonly in the 25-35\% range largely depending upon criteria and methodology [44-55], and these percentages are lower than the calculated values for the AS control group in the present study. The former are based on AS protocols (which have evolved over time) and the published rates have varied depending on whether there was repeat biopsy at baseline (repeat biopsies may exclude subjects with higher baseline Gleason missed on initial biopsy); frequency of biopsies; baseline population characteristics; extent of sampling, and other factors [44-49]. The present study was designed to test treatment effect and (1) did not entail a repeat biopsy prior to enrollment, (2) there were higher core numbers per biopsy (16-core compared to usually $8-12$ in the large series), (3) there were more frequent biopsies ( 5 biopsies by 5 -year time compared to the more usual $\geq 2$ biopsies in the AS protocols, (4) the study had smaller $n$ (not comparable to major AS studies), and (5) included RP surgical pathology results in all patients where this was available after randomization. All of the preceding will give the AS group (and equally so the drug groups in this study) potentially higher (more conservative) numbers of grade progression results [49-55]. Furthermore, all three groups (AS, FT $2.5 \mathrm{mg}$ and $15 \mathrm{mg}$ ) had significant numbers of subjects (e.g,. 33\% of AS group) with two biopsies which were negative but who were excluded from analysis due to early patient withdrawal, and would have qualified as negative under a different criterion of $\geq 2$ biopsies negative. Progression to Gleason $\geq 4+3$ in the AS group in this study (15.4\% at 3 years, biopsy data; $8.7 \%$ if all subjects with $\geq 2$ biopsies are included) was not appreciably higher than the published literature [44-49] which supports that the AS group was representative. A post hoc sensitivity test showed AS progression values in this study are comparable to published AS values if all randomized subjects in all groups with $\geq 2$ biopsies are included (Table 3c, d): AS 25.9\% and 34.6\% at 18 and 36 months, compared to FT $15 \mathrm{mg} 6.5 \%(p=0.0199)$ and $11.8 \%(p=0.033)$, and compared to pooled FT $9.7 \%$ $(p=0.0288)$ and $15.5 \%(p=0.0392)$. PCa progression under AS is complex and depends on many factors (e.g., PSA density, extent of baseline cancer, imaging status, schedule and extent of surveillance, and other factors still being clarified), and even when there are attempts to control these factors, there is still some degree of discordance among different published single-center cohorts [49].

$\mathrm{PCa}$ is the second most common cancer in men (after skin cancer), and is the most common internal cancer in men. With modern era urological diagnostics and therapies, the election for AS approaches has increased. The process of repeated biopsies, examinations, and PSA testing increases patients' anxieties, and there are significant discomforts and potential side effects with the monitoring process (such as biopsy-related urosepsis). Additionally, when conducting AS for Grade Group 1 patients, there does exist the potential for progression of histopathology. Balancing the risks of surgical or radiation interventions vs the risks and anxieties of monitoring and surveillance is a cause of stress and uncertainty for some patients. After 3 years in the reported study (including all patients with $\geq 2$ biopsies and including RP surgical pathology results), $29.6 \%$ of AS patients had interventions with Gleason grade progression $>3+3$, and $34.6 \%$ of patients had Gleason grade $>3+3$. Regardless of whether the subjects were prospectively or retrospectively categorized as low risk or very low risk, all were under AS in the protocol. There were no recorded deaths from PCa in any treated or untreated subjects after 78 months in the trial, which is consistent with the low risk of Grade Group 1 PCa.

Urologists have long recognized the unmet need for prostate cancer treatments that can contribute to improved outcomes for their patients together with reduced side effects and stresses that may significantly impact on quality of life. The goal of a therapy such as FT injectable is to allow for an initial and less toxic treatment for low-risk 
prostate cancer patients, achieving the benefits of molecular ablation with minimal risk of side effects.

\begin{abstract}
Authors' contributions NS: project development, data collection, manuscript writing, and editing. SAK: manuscript writing and editing. RT: project development, data collection, manuscript writing, and editing. RL: data collection and manuscript editing. JB: data collection and manuscript editing. AH: data collection and manuscript editing. SK: data collection and manuscript editing. MB: data collection and manuscript editing. SF: data collection and manuscript editing. KG: data collection and manuscript editing. FS: data collection and manuscript editing. JE: data collection, manuscript writing, and editing.
\end{abstract}

Funding Funded by Nymox Pharmaceutical Corporation

\section{Compliance with ethical standards}

Conflict of interest Author Bailen is consultant for Astellas, Pfizer, Jannsen, Bayer, Dendreon, and Myriad. Author Bidair is consultant for Procept. Author Kaplan is consultant for Astellas, ProVerum Medical, ProArc, Zenflow, Serenity, Allium, Avadel, and Nymox. Author Levin is consultant/speaker for Boston Scientific. Author Shore is consultant/ speaker for Amgen, Astellas, Astra Zeneca, BMS, Bayer, Dendreon, Ferring, Janssen, Pfizer, Merck, Sanofi, Tolmar, and Nymox. Author Tutrone is consultant/speaker for Astella, Pfizer, Dendreon, Bayer, Janssen, and Nymox. Author Tutrone owns stock in Nymox and Myovant Corp. Author Hay owns stock in Nymox.

Statement of human rights All procedures performed in studies involving human participants were in accordance with the ethical standards of the institutional and/or national research committee and with the 1964 Helsinki declaration and its later amendments or comparable ethical standards.

Open Access This article is licensed under a Creative Commons Attribution 4.0 International License, which permits use, sharing, adaptation, distribution and reproduction in any medium or format, as long as you give appropriate credit to the original author(s) and the source, provide a link to the Creative Commons licence, and indicate if changes were made. The images or other third party material in this article are included in the article's Creative Commons licence, unless indicated otherwise in a credit line to the material. If material is not included in the article's Creative Commons licence and your intended use is not permitted by statutory regulation or exceeds the permitted use, you will need to obtain permission directly from the copyright holder. To view a copy of this licence, visit http://creativecommons.org/licenses/by/4.0/.

\section{References}

1. Valerio M, Ahmed HU, Emberton M et al (2014) The role of focal therapy in the management of localised prostate cancer: a systematic review. Eur Urol 66:732-751

2. Wilt TJ, Brawer MK, Jones KM et al (2012) Radical prostatectomy versus observation for localized prostate cancer. N Engl J Med 367:203-213

3. Resnick MJ, Koyama T, Fan KH et al (2013) Long-term functional outcomes after treatment for localized prostate cancer. N Engl J Med 368:436-445
4. Lindner U, Trachtenberg J, Lawrentschuk N (2010) Focal therapy in prostate cancer: modalities, findings and future considerations. Nat Rev Urol 7:562-571

5. Van der Poel H, van den Bergh RCN, Briers E et al (2018) Focal therapy in primary localized prostate cancer: the European association of urology position in 2018. Euro Urol 74:84-91

6. Eggener S, Salomon G, Scardino PT, De la Rosette J, Polascik TJ, Brewster S (2010) Focal therapy for prostate cancer: possibilities and limitations. Eur Urol 58:57-64

7. Van den Bos W, Muller BG, Ahmed H et al (2014) Focal therapy in prostate cancer: international multidisciplinary consensus on trial design. Eur Urol 65:1078-1083

8. Ahmed HU, Akin O, Coleman JA et al (2012) Transatlantic consensus group on active surveillance and focal therapy for prostate cancer. BJU Int 109:1636-1647

9. Bill-Axelson A, Holmberg L, Garmo H et al (2014) Radical prostatectomy or watchful waiting in early prostate cancer. N Engl J Med 370:932-942

10. Ganz PA, Barry JM, Burke W et al (2011) NIH State-of-theScience Conference Statement: role of active surveillance in the management of men with localized prostate cancer. NIH Consens State Sci Statements 28:1-27

11. Valerio M, Cerantola Y, Eggener SE et al (2016) New and established technology in focal ablation of the prostate: a systematic review. Eur Urol 71:17-34

12. Giannarini G, Gandaglia G, Montorsi F, Briganti A (2014) Will focal therapy remain only an attractive illusion for the primary treatment of prostate cancer? J Clin Oncol 32:1299-1301

13. Valerio M, Emberton M, Ahmed HU (2014) Focal therapy will become a standard option for selected men with localized prostate cancer. J Clin Oncol 32:3680-3681

14. Muller BG, van den Bos W, Brausi M et al (2015) Follow-up modalities in focal therapy for prostate cancer: results from a Delphi consensus project. World J Urol 33:1503-1509

15. Donaldson IA, Alonzi R, Barratt D et al (2015) Focal therapy: patients, interventions, and outcomes-a report from a consensus meeting. Eur Urol 67:771-777

16. Ahmed HU, Berge V, Bottomley D et al (2014) Can we deliver randomized trials of focal therapy in prostate cancer? Nat Rev Clin Oncol 11:482-491

17. Hou A, Sullivan K, Crawford D (2009) Targeted focal therapy for prostate cancer: a review. Curr Opin Urol 19:283-289

18. Renard-Penna R, Sanchez-Salas R, Barret E, Cosset et al (2017) Modalités d'évaluation et résultats des traitements ablatifs dans le cancer de la prostate. Prog Urol 27:887-908

19. Mottet N, Bellmunt J, Bolla M et al (2017) EAU-ESTRO-SIOG guidelines on prostate cancer. Part 1: Screening, diagnosis, and local treatment with curative intent. Euro Urol 71:618-629

20. Sanda MG, Cadeddu JA, Kirkby E et al (2018) Clinically localized prostate cancer: AUA/ASTRO/SUO guideline: Part 1: risk stratification, shared decision making, and care options. J Urol 3:683-690

21. Pierorazio PM, Walsh PW, Partin AW et al (2013) Prognostic Gleason grade grouping: data based on the modified Gleason scoring system. BJU Int 111:753-760

22. Epstein JI, Zelefsky MJ, Sjoberg DD et al (2016) A contemporary prostate cancer grading system: a validated alternative to the Gleason score. Eur Urol 69:428-435

23. Epstein JI, Egevad L, Amin MB et al (2016) The 2014 International Society of Urological Pathology (ISUP) consensus conference on Gleason grading of prostatic carcinoma: definition of grading patterns and proposal for a new grading system. Am J Surg Pathol 40:244-252

24. Mayes JM, Mouraviev V, Sun L, Tsivian M, Madden JF, Polascik TJ (2011) Can the conventional sextant prostate biopsy accurately 
predict unilateral prostate cancer in low-risk, localized, prostate cancer? Urol Oncol 29:166-170

25. Berg KD, Toft BG, Roder MA, Brasso K, Vainer B, Iversen P (2013) Is it possible to predict low-volume and insignificant prostate cancer by core needle biopsies? APMIS 121:257-265

26. Gallina A, Maccagnano C, Suardi N et al (2012) Unilateral positive biopsies in low risk prostate cancer patients diagnosed with extended transrectal ultrasound-guided biopsy schemes do not predict unilateral prostate cancer at radical prostatectomy. BJU Int 110:E64-E68

27. Washington SL, Bonham M, Whitson JM, Cowan JE, Carroll PR (2012) Transrectal ultrasonography-guided biopsy does not reliably identify dominant cancer location in men with low-risk prostate cancer. BJU Int 110:50-55

28. Katz B, Srougi M, Dall'Oglio M et al (2012) Are we able to correctly identify prostate cancer patients who could be adequately treated by focal therapy? Urol Oncol 30:794-797

29. Tsivian M, Moreira DM, Sun L et al (2012) Biopsy accuracy in identifying unilateral prostate cancer depends on prostate weight. Urol Oncol 30:21-25

30. Bott SR, Ahmed HU, Hindley RG, Abdul-Rahman A, Freeman A, Emberton M (2010) The index lesion and focal therapy: an analysis of the pathological characteristics of prostate cancer. BJU Int 106:1607-1611

31. Algaba F, Montironi R (2010) Impact of prostate cancer multifocality on its biology and treatment. J Endourol 24:799-804

32. Ahmed HU (2009) The index lesion and the origin of prostate cancer. N Engl J Med 361:1704-1706

33. Ahmed HU, Arya M, Freeman A, Emberton M (2012) Do lowgrade and low-volume prostate cancers bear the hallmarks of malignancy? Lancet Oncol 13:e509-e517

34. Hankey B, Feuer E, Clegg L et al (1999) Cancer surveillance series: interpreting trends in prostate cancer-Part I: Evidence of the effects of screening in recent prostate cancer incidence, mortality, and survival rates. J Natl Cancer Inst 91:1017-1024

35. Albertsen P, Hanley J, Gleason D, Barry M (1998) Competing risk analysis of med aged 55 to 74 years at diagnosis managed conservatively for clinically localized prostate cancer. JAMA 280:975-980

36. Albertsen P, Hanley J, Fine J (2005) 20-year outcomes following conservative management of clinically localized prostate cancer. JAMA 293:2095-2101

37. Shore N, Tutrone R, Roehrborn C (2019) Efficacy and safety of fexapotide triflutate in outpatient medical treatment of male lower urinary tract symptoms associated with benign prostatic hyperplasia. Ther Adv Urol 11:1-16

38. Shore N, Tutrone R, Efros M et al (2018) Fexapotide triflutate: results of long-term safety and efficacy trials of a novel injectable therapy for symptomatic prostate enlargement. World J Urol 36:801-809

39. Shore N, Cowan B (2011) The potential for NX-1207 in benign prostatic hyperplasia: an update for clinicians. Ther Adv Chronic Dis 2:377-383

40. Shore N (2010) NX-1207: a novel investigational drug for the treatment of benign prostatic hyperplasia. Expert Opin Invest Drugs 19:305-310

41. Tutrone R, Bidair M, Grunberger I et al (2018) Phase 3 clinical studies and biology of fexapotide triflutate office injectable for BPH. In: Presented at the American Urological Association annual meeting, 20 May 2018, San Francisco
42. Tutrone R, Goldberg K, Grunberger I et al (2017) Fexapotide triflutate: first in class injectable for BPH. In: Presented at the American Urological Association New York section annual meeting, 6 Novr 2017, Havana

43. Hay A, Bidair M, Bailen J (2017) Fexapotide triflutate: a safe and effective injectable for BPH. In: Presented at the American Urological Associates south central annual meeting, 27 Nov 2017, Naples

44. Inoue LYT, Lin DW, Newcomb LF et al (2018) Comparative analysis of biopsy upgrading in four prostate cancer active surveillance cohorts. Ann Intern Med 168:1-9

45. Nassiri N, Margolis DJ, Natarajan S et al (2017) Targeted biopsy to detect Gleason score upgrading during active surveillance for men with low vs intermediate-risk prostate cancer. J Urol 197:632-639

46. Jain S, Loblaw A, Vesprini D et al (2015) Gleason upgrading with time in a large prostate cancer active surveillance cohort. J Urol 194:79-84

47. Masic S, Cowan J, Washington SL et al (2018) Effects of Gleason grade on outcomes during active surveillance for prostate cancer. Eur Urol Oncol 1:386-394

48. Bul M, Zhu X, Valdagni R et al (2013) Active surveillance for low-risk prostate cancer worldwide: the PRIAS study. Eurol Urol 63:597-603

49. Welty CJ, Cooperberg MR, Carroll P (2014) Meaningful end points and outcomes in men on active surveillance for early-stage prostate cancer. Curr Opin Urol 24:288-292

50. Bul M, Zhu X, Rannikko A et al (2012) Radical prostatectomy for low-risk prostate cancer following initial active surveillance: results from a prospective observational study. Eur Urol 62:195-200

51. Conti SL, Dall'era M, Fradet V et al (2009) Pathologic outcomes of candidates for active surveillance of prostate cancer. J Urol 181:1628-1633

52. Epstein JI, Feng Z, Trock BJ, Pierorazio PM (2012) Upgrading and downgrading of prostate cancer from biopsy to radical prostatectomy: incidence and predictive factors using the modified Gleason grading system and factoring in tertiary grades. Eur Urol 61:1019-1024

53. Seisen T, Roudot-Thoraval F, Bosset PO et al (2015) Predicting the risk of harboring high-grade disease for patients diagnosed with prostate cancer scored as Gleason $\leq 6$ on biopsy cores. World J Urol 33:787-792

54. Vellekoop A, Loeb S, Folkvaljon Y, Stattin P (2014) Population based study of predictors of adverse pathology among candidates for active surveillance with Gleason 6 prostate cancer. J Urol 191:350-357

55. Suardi N, Capitanio U, Chun FK et al (2008) Currently used criteria for active surveillance in men with low-risk prostate cancer: an analysis of pathologic features. Cancer 113:2068-2072

Publisher's Note Springer Nature remains neutral with regard to jurisdictional claims in published maps and institutional affiliations. 